\title{
ANALYTIC SOLUTION OF TRANSCENDENTAL EQUATIONS
}

\author{
HENRYK GÓRECKI \\ Faculty of Informatics \\ Higher School of Informatics, ul. Rzgowska 17a, 93-008 Łódź, Poland \\ e-mail: head@nova.ia.agh.edu.pl
}

\begin{abstract}
A decomposition technique of the solution of an $n$-th order linear differential equation into a set of solutions of 2-nd order linear differential equations is presented.
\end{abstract}

Keywords: transcendental equations, zeros, extrema.

\section{Introduction}

Let us consider the differential equation determining the transient error in a linear control system of the $n$-th order with lumped and constant parameters $a_{i}, i=1, \ldots, n$ :

$\frac{\mathrm{d}^{n} x(t)}{\mathrm{d} t^{n}}+a_{1} \frac{\mathrm{d}^{n-1} x(t)}{\mathrm{d} t^{n-1}}+\cdots+a_{n-1} \frac{\mathrm{d} x(t)}{\mathrm{d} t}+a_{n} x(t)=0$,

with the initial conditions which, in general, are different from zero:

$$
x^{(i-1)}(0)=c_{i} \neq 0 \quad \text { for } \quad i=1,2, \ldots, n .
$$

The solution of Eqn. (1) takes the following form:

$$
x(t)=\sum_{k=1}^{n} A_{k} e^{s_{k} t}
$$

where $s_{k}$ are the simple roots of the characteristic equation

$$
s^{n}+a_{1} s^{n-1}+\cdots+a_{n-1} s+a_{n}=0 .
$$

In order to obtain an explicit form for $A_{k}$, we need higher derivatives of $x(t)$ :

$$
\frac{\mathrm{d}^{p} x(t)}{\mathrm{d} t^{p}}=\sum_{k=1}^{n} s_{k}^{p} A_{k} e^{s_{k} t}, \quad p=1,2, \ldots, n-1 .
$$

The formulae (2) and (4) represent a system of $n$ linear equations with respect to unknown terms $A_{k} e^{s_{k} t}$. Its ma- trix of coefficients is the Vandermonde matrix

$$
\left(\begin{array}{cccc}
1 & 1 & \ldots & 1 \\
s_{1} & s_{2} & \ldots & s_{n} \\
\vdots & \vdots & & \vdots \\
s_{1}^{n-1} & s_{2}^{n-1} & \ldots & s_{n}^{n-1}
\end{array}\right) .
$$

According to the assumption that $s_{i} \neq s_{j}$ for $i \neq j$, the matrix (5) has an inverse and the system (2) and (4) can be solved. We denote by $V$ the Vandermonde determinant of the matrix (5) and by $V_{k}$ the Vandermonde determinant of order $(n-1)$ of the variables $s_{1}, \ldots, s_{k-1}, s_{k+1}, \ldots, s_{n}$.

We also denote by $\Phi_{r}^{(k)}$ the fundamental symmetric function of the $r$-th order of $(n-1)$ variables $s_{1}, \ldots$, $s_{k-1}, s_{k+1}, \ldots, s_{n}$ for $r=0,1, \ldots, n-1$ :

$$
\begin{aligned}
\Phi_{0}^{(k)}= & 1, \\
\Phi_{1}^{(k)}= & s_{1}+s_{2}+\cdots+s_{k-1}+s_{k+1} \cdots+s_{n} \\
= & -a_{1}-s_{k}, \\
\Phi_{2}^{(k)}= & s_{1} s_{2}+s_{1} s_{3}+\cdots+s_{1} s_{k-1}+s_{1} s_{k+1} \\
& \cdots+s_{2} s_{3}+\cdots+s_{2} s_{k-1}+s_{2} s_{k+1} \\
& \cdots+s_{2} s_{n}+\cdots \\
= & a_{2}-s_{1} s_{k}-s_{2} s_{k}-\cdots-s_{n} s_{k}, \\
\Phi_{n}^{(k)}= & \prod_{i=1, i \neq k}^{n} s_{i}=(-1)^{n} \frac{a_{n}}{s_{k}} .
\end{aligned}
$$

It can be shown that the elements of the matrix inverse to the matrix (5) have the form

$$
\alpha_{i k}=\frac{(-1)^{i+k}}{V} \cdot \Phi_{n-k}^{(i)} V_{k} .
$$


The solution of the system (2) and (4) is as follows:

$$
\begin{aligned}
A_{k} e^{s_{k} t} & =\sum_{j=1}^{n} \alpha_{k j} x^{(j-1)}(t) \\
& =\sum_{j=1}^{n} \frac{(-1)^{k+j}}{V} \cdot \Phi_{n-j}^{(k)} V_{k} x^{(j-1)}(t)
\end{aligned}
$$

or

$$
\begin{array}{r}
A_{k} e^{s_{k} t}=\frac{(-1)^{k} V_{k}}{V} \sum_{j=1}^{n}(-1)^{j} \Phi_{n-j}^{(k)} x^{(j-1)}(t), \\
k=1,2, \ldots, n .
\end{array}
$$

For $t=0$, we know $x^{(j-1)}(0)=c_{j}$, and the substitution $t=0$ into 9 gives

$$
A_{k}=\frac{(-1)^{k} V_{k}}{V} \sum_{j=1}^{n}(-1)^{j} \Phi_{n-j}^{(k)} c_{j} .
$$

Using the relation (6), we can formulate the following theorem.

Theorem 1. The explicit form of the coefficient $A_{1}$ is as follows:

$$
\begin{aligned}
A_{1}= & \frac{c_{n}-\left(\sum_{j=1}^{n} s_{j}\right) c_{n-1}+\left(\sum_{i, j \neq i \neq 1}^{n} s_{j} s_{i}\right) c_{n-2}}{\left(s_{n}-s_{1}\right)\left(s_{n-1}-s_{1}\right) \ldots\left(s_{2}-s_{1}\right)} \\
- & \frac{\left(\sum_{i, j, k \neq 1}^{n} s_{i} s_{j} s_{k}\right) c_{n-3}+\ldots}{\left(s_{n}-s_{1}\right)\left(s_{n-1}-s_{1}\right) \ldots\left(s_{2}-s_{1}\right)} \\
+ & \frac{\ldots+(-1)^{n-1} \prod_{i \neq 1}^{n} s_{i} c_{1}}{\left(s_{n}-s_{1}\right)\left(s_{n-1}-s_{1}\right) \ldots\left(s_{2}-s_{1}\right)} .
\end{aligned}
$$

Then the coefficients $A_{2}, A_{3}, \ldots, A_{n}$ can be obtained by the sequential change of the indices of $s_{i}$ according to the scheme

$$
s_{1} \longrightarrow s_{2} \longrightarrow s_{3} \longrightarrow \ldots \longrightarrow s_{n-1} \longrightarrow s_{n} \longrightarrow s_{1} \text {. }
$$

Example 1. The solution of the 3-rd order equation is as follows:

$$
x(t)=A_{1} e^{s_{1} t}+A_{2} e^{s_{2} t}+A_{3} e^{s_{3} t} .
$$

The coefficient

$$
A_{1}=\frac{(-1) V_{1}}{V} \sum_{j=1}^{3}(-1)^{j} \Phi_{3-j}^{(1)} c_{j},
$$

where

$$
\begin{aligned}
V_{1} & =\left|\begin{array}{cc}
1 & 1 \\
s_{2} & s_{3}
\end{array}\right|=s_{3}-s_{2}, \\
V & =\left|\begin{array}{ccc}
1 & 1 & 1 \\
s_{1} & s_{2} & s_{3} \\
s_{1}^{2} & s_{2}^{2} & s_{3}^{2}
\end{array}\right|=\left(s_{2}-s_{1}\right)\left(s_{3}-s_{2}\right)\left(s_{3}-s_{1}\right), \\
\Phi_{0}^{(1)} & =1, \quad \Phi_{1}^{(1)}=s_{2}+s_{3}, \quad \Phi_{2}^{(1)}=s_{2} s_{3},
\end{aligned}
$$

$$
\begin{aligned}
A_{1}= & \frac{(-1)\left(s_{3}-s_{2}\right)}{\left(s_{2}-s_{1}\right)\left(s_{3}-s_{2}\right)\left(s_{3}-s_{1}\right)} \\
& \cdot\left[(-1) \Phi_{2}^{(1)} c_{1}+\Phi_{1}^{(1)} c_{2}-\Phi_{0}^{(1)} c_{3}\right] \\
= & \frac{(-1)\left[-s_{2} s_{3} c_{1}+\left(s_{2}+s_{3}\right) c_{2}-c_{3}\right]}{\left(s_{2}-s_{1}\right)\left(s_{3}-s_{1}\right)} \\
= & \frac{c_{3}-\left(s_{2}+s_{3}\right) c_{2}+s_{2} s_{3} c_{1}}{\left(s_{2}-s_{1}\right)\left(s_{3}-s_{1}\right)}, \\
A_{2}= & \frac{c_{3}-\left(s_{3}+s_{1}\right) c_{2}+s_{3} s_{1} c_{1}}{\left(s_{3}-s_{2}\right)\left(s_{1}-s_{2}\right)}, \\
A_{3}= & \frac{c_{3}-\left(s_{1}+s_{2}\right) c_{2}+s_{1} s_{2} c_{1}}{\left(s_{1}-s_{3}\right)\left(s_{2}-s_{3}\right)} .
\end{aligned}
$$

After the substitution of (10) into (9), we obtain

$$
\begin{aligned}
e^{s_{k} t} \frac{(-1)^{k} V_{k}}{V} \sum_{j=1}^{n}(-1)^{j} \Phi_{n-j}^{(k)} x^{(j-1)}(0) & \\
& =\frac{(-1)^{k} V_{k}}{V} \sum_{j=1}^{n}(-1)^{j} \Phi_{n-j}^{(k)} x^{(j-1)}(t)
\end{aligned}
$$

and, finally, for $k=1,2, \ldots, n$, we have

$$
e^{s_{k} t} \sum_{j=1}^{n}(-1)^{j} \Phi_{n-j}^{(k)} c_{j}=\sum_{j=1}^{n}(-1)^{j} \Phi_{n-j}^{(k)} x^{(j-1)}(t) .
$$

Premultiplying both sides of 12 and using Viete's relations between the roots $s_{i}$ and the coefficient $a_{1}$ of the characteristic equation,

$$
\sum_{k=1}^{n} s_{k}=-a_{1}
$$

we obtain the main result formulated as Theorem 2

Theorem 2. (Górecki and Turowicz, 1968) The relation between cofficients $a_{i}, i=1,2, \ldots, n$, the initial values $c_{j}, j=1,2, \ldots, n$ and solutions $x^{(j)}(t)$ is as follows:

$$
\begin{aligned}
e^{-a_{1} t} \prod_{k=1}^{n} \sum_{j=1}^{n}(-1)^{j} \Phi_{n-j}^{(k)} c_{j} & \\
& =\prod_{k=1}^{n} \sum_{j=1}^{n}(-1)^{j} \Phi_{n-j}^{(k)} x^{(j-1)}(t) .
\end{aligned}
$$


Both the sides of Eqn. (2) are composed of symmetric polynomials of variables $s_{1}, \ldots, s_{n}$. Accordingly, it is possible to express these terms as polynomials of the coefficiets $a_{1}, \ldots, a_{n}$. Using Vieta's relations, it is possible to replace the roots $s_{k}$ by the coefficients $a_{i}$ and to avoid calculating the roots by solving algebraic equations.

Example 2. For $n=3$, we have

$$
\begin{aligned}
& e^{-a_{1} t}\left\{a_{3}^{2} c_{1}^{3}+2 a_{2} a_{3} c_{2} c_{1}^{2}+\left(a_{1} a_{3}+a_{2}^{2}\right) c_{2}^{2} c_{1}\right. \\
& +\left(a_{1} a_{2}-a_{3}\right) c_{2}^{3}+\left(a_{1} a_{2}+3 a_{3}\right) c_{1} c_{2} c_{3}+a_{1} a_{3} c_{1}^{2} c_{3} \\
& \left.+a_{2} c_{1} c_{3}^{2}+\left(a_{1}^{2}+a_{2}\right) c_{2}^{2} c_{3}+2 a_{1} c_{2} c_{3}^{2}+c_{3}^{3}\right\} \\
& =a_{3}^{2}[x(t)]^{3}+2 a_{2} a_{3} x^{(1)}(t)[x(t)]^{2}+\left(a_{1} a_{3}+a_{2}^{2}\right) \\
& \cdot\left[x^{(1)}(t)\right]^{2} x(t)+\left(a_{1} a_{2}-a_{3}\right)\left[x^{(1)}(t)\right]^{3} \\
& +\left(a_{1} a_{2}+3 a_{3}\right) x(t) x^{(1)}(t) x^{(2)}(t) \\
& +a_{1} a_{3}[x(t)]^{2} x^{(2)}(t)+a_{2} x(t)\left[x^{(2)}(t)\right]^{2} \\
& +\left(a_{1}^{2}+a_{2}\right)\left[x^{(1)}(t)\right]^{2} x^{(2)}(t)+2 a_{1} x^{(1)}(t) \\
& \cdot\left[x^{(2)}(t)\right]^{2}+\left[x^{(2)}(t)\right]^{3},
\end{aligned}
$$

where

$$
\begin{aligned}
& a_{1}=-\left(s_{1}+s_{2}+s_{3}\right), \\
& a_{2}=s_{1} s_{2}+s_{1} s_{3}+s_{2} s_{3}, \\
& a_{3}=-s_{1} s_{2} s_{3} .
\end{aligned}
$$

Example 3. For $n=4$, similarly

$$
\begin{array}{rl}
e^{-a_{1}} & t\left[a_{4}^{3} c_{1}^{4}+3 a_{3} a_{4}^{2} c_{1}^{3} c_{2}+2 a_{2} a_{4}^{2} c_{1}^{3} c_{3}+a_{1} a_{4}^{2} c_{1}^{3} c_{4}\right. \\
& +\left(3 a_{3}^{2} a_{2} a_{4}\right) a_{4} c_{1}^{2} c_{2}^{2}+\left(4 a_{2} a_{3}+3 a_{1} a_{4}\right) a_{4} c_{1}^{2} c_{2} c_{3} \\
& +2\left(a_{1} a_{3}+2 a_{4}\right) a_{4} c_{1}^{2} c_{2} c_{4} \\
& +\left(a_{2}^{2}+a_{1} a_{3}+2 a_{4}\right) a_{4} c_{1}^{2} c_{3}^{2} \\
& +\left(a_{1} a_{2}+3 a_{3}\right) a_{4} c_{1}^{2} c_{3} c_{4}+a_{2} a_{4} c_{1}^{2} c_{4}^{2} \\
& +\left(a_{3}^{3}+2 a_{2} a_{3} a_{4}-a_{1} a_{4}^{2}\right) c_{1} c_{2}^{3}+2\left(a_{2} a_{3}^{2}+a_{2}^{2} a_{4}\right. \\
& \left.+2 a_{1} a_{3} a_{4}-2 a_{4}^{2}\right) c_{1} c_{2}^{2} c_{3} \\
& +\left(a_{1} a_{3}^{2}+a_{1} a_{2} a_{4}+5 a_{3} a_{4}\right) c_{1} c_{2}^{2} c_{4} \\
& +\left(a_{2}^{2} a_{3}+a_{1} a_{3}^{2}+5 a_{1} a_{2} a_{4}-a_{3} a_{4}\right) c_{1} c_{2} c_{3}^{2} \\
& +\left(a_{1} a_{2} a_{3}+3 a_{1}^{2} a_{4}+3 a_{3}^{2}+4 a_{2} a_{4}\right) c_{1} c_{2} c_{3} c_{4} \\
& +\left(a_{2} a_{3}+3 a_{1} a_{4}\right) c_{1} c_{2} c_{4}^{2} \\
& +\left(a_{1} a_{2} a_{3}+a_{1}^{2} a_{4}-a_{3}^{2}+2 a_{2} a_{4}\right) c_{1} c_{3}^{3} \\
& +\left(a_{1}^{2} a_{3}+a_{2} a_{3}+5 a_{1} a_{4}\right) c_{1} c_{3}^{2} c_{4} \\
& +2\left(a_{1} a_{3}+2 a_{4}\right) c_{1} c_{3} c_{4}^{2}+a_{3} c_{1} c_{4}^{3} \\
& +\left(a_{2} a_{3}^{2}-a_{1} a_{3} a_{4}+a_{4}^{2}\right) c_{2}^{4} \\
& +\left(2 a_{2}^{2} a_{3}+a_{1} a_{3}^{2}-a_{1} a_{2} a_{4}-a_{3} a_{4}\right) c_{2}^{3} c_{3} \\
& +\left(a_{1} a_{2} a_{3}-a_{1}^{2} a_{4}+a_{3}^{2}-2 a_{2} a_{4}\right) c_{2}^{3} c_{4} \\
& +a_{2}\left(a_{2}^{2}+3 a_{1} a_{3}-3 a_{4}\right) c_{2}^{2} c_{3}^{2} \\
&
\end{array}
$$

(a)

$+\left(a_{1} a_{2}^{2}+a_{1}^{2} a_{3}+5 a_{2} a_{3}-a_{1} a_{4}\right) c_{2}^{2} c_{3} c_{4}$

$+\left(a_{2}^{2}+a_{1} a_{3}+2 a_{4}\right) c_{2}^{2} c_{4}^{2}$

$+\left(2 a_{1} a_{2}^{2}+a_{1}^{2} a_{3}-a_{2} a_{3}-a_{1} a_{4}\right) c_{2} c_{3}^{3}$

$+2\left(a_{1}^{2} a_{2}+a_{2}^{2}+2 a_{1} a_{3}-2 a_{4}\right) c_{2} c_{3}^{2} c_{4}$

$+\left(4 a_{1} a_{2}+3 a_{3}\right) c_{2} c_{3} c_{4}^{2}+2 a_{2} c_{2} c_{4}^{3}$

$\left(a_{1}^{2} a_{2}-a_{1} a_{3}+a_{4}\right) c_{3}^{4}+\left(a_{1}^{3}+2 a_{1} a_{2}-a_{3}\right) c_{3}^{3} c_{4}$

$\left.+\left(3 a_{1}^{2}+a_{2}\right) c_{3}^{2} c_{4}^{2}+3 a_{1} c_{3} c_{4}^{3}+c_{4}^{4}\right]$

$=a_{4}^{3} x(t)^{4}+3 a_{3} a_{4}^{2} x(t)^{3} x^{(1)}(t)$

$+2 a_{2} a_{4}^{2} x(t)^{3} x^{(2)}(t)+a_{1} a_{4}^{2} x(t)^{3} x^{(3)}(t)$

$+\left(3 a_{3}^{2}+a_{2} a_{4}\right) a_{4} x(t)^{2}\left[x^{(1))}(t)\right]^{2}$

$+\left(4 a_{2} a_{3}+3 a_{1} a_{4}\right) a_{4} x(t)^{2} x^{(1)}(t) x^{(2)}(t)$

$+2\left(a_{1} a_{3}+2 a_{4}\right) a_{4} x(t)^{2} x^{(1)}(t) x^{(3)}(t)$

$+\left(a_{2}^{2}+a_{1} a_{3}+2 a_{4}\right) a_{4} x(t)^{2}\left[x^{(2)}(t)\right]^{2}$

$+\left(a_{1} a_{2}+3 a_{3}\right) a_{4} x(t)^{2} x^{(2)}(t) x^{(3)}(t)$

$+a_{2} a_{4} x(t)^{2}\left[x^{(3)}(t)\right]^{2}$

$+\left(a_{3}^{3}+2 a_{2} a_{3} a_{4}-a_{1} a_{4}^{2}\right) x(t)\left[x^{(1)}(t)\right]^{3}$

$+2\left(a_{2} a_{3}^{2}+a_{2}^{2} a_{4}+2 a_{1} a_{3} a_{4}-2 a_{4}^{2}\right) x(t)$

$\cdot\left[x^{(1)}(t)\right]^{2} x^{(2)}(t)+\left(a_{1} a_{3}^{2}+a_{1} a_{2} a_{4}+5 a_{3} a_{4}\right)$

$\cdot x(t)\left[x^{(1)}(t)\right]^{2} x^{(3)}(t)$

$+\left(a_{2}^{2} a_{3}+a_{1} a_{3}^{2}+5 a_{1} a_{2} a_{4}-a_{3} a_{4}\right) x(t) x^{(1)}(t)$

$\cdot\left[x^{(2)}(t)\right]^{2}$

$+\left(a_{1} a_{2} a_{3}+3 a_{1}^{2} a_{4}+3 a_{3}^{2}+4 a_{2} a_{4}\right)$

$\cdot x(t) x^{(1)}(t) x^{(2)}(t) x^{(3)}(t)$

$+\left(a_{2} a_{3}+3 a_{1} a_{4}\right) x(t) x^{(1)}(t)\left[x^{(3)}(t)\right]^{2}$

$+\left(a_{1} a_{2} a_{3}+a_{1}^{2} a_{4}-a_{3}^{2}+2 a_{2} a_{4}\right) x(t)\left[x^{(2)}(t)\right]^{3}$

$+\left(a_{1}^{2} a_{3}+a_{2} a_{3}+5 a_{1} a_{4}\right) x(t)\left[x^{(2)}(t)\right]^{2} x^{(3)}(t)$

$+2\left(a_{1} a_{3}+2 a_{4}\right) x(t) x^{(2)}(t)\left[x^{(3)}(t)\right]^{2}$

$+a_{3} x(t)\left[x^{(3)}(t)\right]^{3}$

$+\left(a_{2} a_{3}^{2}-a_{1} a_{3} a_{4}+a_{4}^{2}\right)\left[x^{(1)}(t)\right]^{4}$

$\left(2 a_{2}^{2} a_{3}+a_{1} a_{3}^{2}-a_{1} a_{2} a_{4}-a_{3} a_{4}\right)\left[x^{(1)}(t)\right]^{3} x^{(2)}(t)$

$+\left(a_{1} a_{2} a_{3}-a_{1}^{2} a_{4}+a_{3}^{2}+2 a_{2} a_{4}\right)\left[x^{(1)}(t)\right]^{3} x^{(3)}(t)$

$+a_{2}\left(a_{2}^{2}+3 a_{1} a_{3}-3 a_{4}\right)\left[x^{(1)}(t)\right]^{2}\left[x^{(2)}(t)\right]^{2}$

$+\cdots+\left(a_{1} a_{2}^{2}+a_{1}^{2} a_{3}+5 a_{2} a_{3}-a_{1} a_{4}\right)\left[x^{(1)}(t)\right]^{2}$

$\cdot x^{(2)}(t) x^{(3)}(t)$

$+\left(a_{2}^{2}+a_{1} a_{3}+2 a_{4}\right)\left[x^{(1)}(t)\right]^{2}\left[x^{(3)}(t)\right]^{2}$

$+\left(2 a_{1} a_{2}^{2}+a_{1}^{2} a_{3}-a_{2} a_{3}-a_{1} a_{4}\right) x^{(1)}(t)\left[x^{(2)}(t)\right]^{3}$

$2\left(a_{1}^{2} a_{2}+a_{2}^{2}+2 a_{1} a_{3}-2 a_{4}\right) x^{(1)}(t)\left[x^{(2)}(t)\right]^{2} x^{(3)}(t)$ 


$$
\begin{aligned}
& +\left(4 a_{1} a_{2}+3 a_{3}\right) x^{(1)}(t) x^{(2)}(t)\left[x^{(3)}(t)\right]^{2} \\
& +2 a_{2} c_{2}\left[x^{(3)}(t)\right]^{3} \\
& +\left(a_{1}^{2} a_{2}-a_{1} a_{3}+a_{4}\right)\left[x^{(2)}(t)\right]^{4} \\
& +\left(a_{1}^{3}+2 a_{1} a_{2}-a_{3}\right)\left[x^{(2)}(t)\right]^{3} x^{(3)}(t) \\
& +\left(3 a_{1}^{2}+a_{2}\right)\left[x^{(2)}(t)\right]^{2}\left[x^{(3)}(t)\right]^{2} \\
& +3 a_{1} x^{(2)}(t)\left[x^{(3)}(t)\right]^{3}+\left[x^{(3)}(t)\right]^{4} .
\end{aligned}
$$

\section{Analytical method of determining zeroes and extremal values of the solution $x(t)$ described by the relation (2)}

2.1. Basic results. The general relation analogous to the formulae (15) or (16) for the equation of the $n$-th order is very complicated. For that reason, we illustrate the method on examples of equations of the 3 -rd and 4 -th orders. We assume that at the extremal point $t_{e}$, or at the zero $t_{0}$ of the solution (2), the second derivative $\mathrm{d}^{2} x / \mathrm{d} t^{2} \neq 0$. We can write the relation (15) in the following form:

$$
\begin{aligned}
& {\left[x^{(2)}\right]^{3}\left\{\left[\frac{x}{x^{(2)}}\right]^{3} a_{3}^{2}+\left(2 a_{2} a_{3} \frac{x^{(1)}}{x^{(2)}}+a_{1} a_{3}\right)\left[\frac{x}{x^{(2)}}\right]^{2}\right.} \\
&+ {\left[\left(a_{1} a_{3}+a_{2}^{2}\right)\left(\frac{x^{(1)}}{x^{(2)}}\right)^{2}+\left(a_{1} a_{2}+3 a_{3}\right) \frac{x^{(1)}}{x^{(2)}}+a_{2}\right] \frac{x}{x^{(2)}} } \\
&+ {\left[\left(a_{1} a_{2}-a_{3}\right)\left(\frac{x^{(1)}}{x^{(2)}}\right)^{3}+\left(a_{1}^{2}+a_{2}\right)\left(\frac{x^{(1)}}{x^{(2)}}\right)^{2}\right.} \\
&+\left.\left.2 a_{1} \frac{x^{(1)}}{x^{(2)}}+1\right]\right\} \\
&= e^{-a_{1} t} c_{3}^{3}\left\{a_{3}^{2}\left(\frac{c_{1}}{c_{2}}\right)^{3}+\left(2 a_{2} a_{3} \frac{c_{2}}{c_{3}}+a_{1} a_{3}\right)\left(\frac{c_{1}}{c_{3}}\right)^{2}\right. \\
&+\left[\left(a_{1} a_{3}+a_{2}^{2}\right)\left(\frac{c_{2}}{c_{3}}\right)^{2}+\left(a_{1} a_{2}+3 a_{3}\right) \frac{c_{2}}{c_{3}}+a_{2}\right] \frac{c_{1}}{c_{3}} \\
&+\left[\left(a_{1} a_{2}-a_{3}\right)\left(\frac{c_{2}}{c_{3}}\right)^{3}+\left(a_{1}^{2}+a_{2}\right)\left(\frac{c_{2}}{c_{3}}\right)^{2}\right. \\
&\left.\left.+2 a_{1} \frac{c_{2}}{c_{3}}+1\right]\right\} .
\end{aligned}
$$

Setting

$$
\begin{gathered}
\frac{x}{x^{(2)}}=\frac{c_{1}}{c_{3}}=u, \\
\frac{x^{(1)}}{x^{(2)}}=\frac{c_{2}}{c_{3}}=v,
\end{gathered}
$$

we can write the relations (17) in the following form:

$$
\begin{aligned}
& \left\{\left[x^{(2)}\right]^{3}-e^{-a_{1} t} c_{3}^{3}\right\} \\
& \cdot\left\{\left[a_{3}^{2} u^{3}+\left(2 a_{2} a_{3} v+a_{1} a_{3}\right) u^{2}\right.\right. \\
& \quad+\left[\left(a_{1} a_{3}+a_{2}^{2}\right) v+a_{2}\right] u \\
& \left.\quad+\left[\left(a_{1} a_{2}-a_{3}\right) v^{3}+\left(a_{1}^{2}+a_{2}\right) v^{2}+2 a_{1} v+1\right]\right\}=0 .
\end{aligned}
$$

If we assume that $c_{2}=0$, then from (19) we have $x^{(1)}\left(t_{e}\right)=0$ and $v=0$. It is a necessary condition for extremum. In this case the equation (20) has a simple form:

$$
\left\{\left[x^{(2)}\right]^{3}-e^{-a_{1} t_{e}} c_{3}^{3}\right\}\left[a_{3}^{2} u^{3}+a_{1} a_{3} u^{2}+a_{2} u+1\right]=0 .
$$

If we assume $c_{1}=0$, then from (18) we obtain that $x\left(t_{0}\right)=0$ and $u=0$. It is a necessary condition for $x(t)$ to be zero. In this case, Eqn. (20) has the following form:

$$
\begin{aligned}
& \left\{\left[x^{(2)}\right]^{3}-e^{-a_{1} t_{0}} c_{3}^{3}\right\} \\
& \left.\cdot\left[a_{1} a_{2}-a_{3}\right) v^{3}+\left(a_{1}^{2}+a_{2}\right) v^{2}+2 a_{1} v+1\right]=0 .
\end{aligned}
$$

It is possible to find the relations between the roots of the equation

$$
a_{3}^{2} u^{3}+a_{1} a_{3} u^{2}+a_{2} u+1=0,
$$

and the roots $s_{1}, s_{2}$ and $s_{3}$ of the characteristic equation

$$
s^{3}+a_{1} s^{2}+a_{2} s+a_{3}=0 .
$$

Setting

$$
u=\frac{y}{\sqrt[3]{a_{3}^{2}}}
$$

in Eqn. 23), we obtain the following:

$$
y^{3}+\frac{a_{1}}{\sqrt[3]{a_{3}}} y^{2}+\frac{a_{2}}{\sqrt[3]{a_{3}^{2}}} y+1=0 .
$$

Similarly, setting

$$
s=\sqrt[3]{a_{3}} z
$$

in Eqn. (24), we obtain that

$$
z^{3}+\frac{a_{1}}{\sqrt[3]{a_{3}}} z^{2}+\frac{a_{2}}{\sqrt[3]{a_{3}^{2}}} z+1=0 .
$$

Equations (26) and (28) are identical. As a result, we have that

$$
y=z \quad \text { or } \quad \sqrt[3]{a_{3}^{2}} u=\frac{s}{\sqrt[3]{a_{3}}} .
$$

Finally, from (29), we conclude that

$$
u=\frac{s}{a_{3}} .
$$

Returning to (19), we find that, if $x^{(1)}=c_{2}=0$, at the extremum point $t_{e}$ the following relations hold:

$$
\frac{x\left(t_{e}\right)}{x^{(2)}\left(t_{e}\right)}=\frac{c_{1}}{c_{3}}=\frac{s_{i}}{a_{3}}, \quad i=1,2,3 .
$$

Taking into account in (31) that $a_{3}=-s_{1} s_{2} s_{3}$, we finally obtain that

$$
\begin{aligned}
& \left.\begin{array}{l}
\frac{x\left(t_{e}\right)}{x^{(2)}\left(t_{e}\right)}=\frac{c_{1}}{c_{3}}=-\frac{1}{s_{2} s_{3}} \quad \text { or } \\
\frac{x\left(t_{e}\right)}{x^{(2)}\left(t_{e}\right)}=\frac{c_{1}}{c_{3}}=-\frac{1}{s_{3} s_{1}} \quad \text { or }
\end{array}\right\} \\
& \frac{x\left(t_{e}\right)}{x^{(2)}\left(t_{e}\right)}=\frac{c_{1}}{c_{3}}=-\frac{1}{s_{1} s_{2}} . \quad
\end{aligned}
$$


Theorem 3. From the relations (32) it is possible to determine extrema (if they exist) using the relations

$$
\left.\begin{array}{l}
s_{2} s_{3} x\left(t_{e}\right)+x^{(2)}\left(t_{e}\right)=0, \\
s_{3} s_{1} x\left(t_{e}\right)+x^{(2)}\left(t_{e}\right)=0, \\
s_{1} s_{2} x\left(t_{e}\right)+x^{(2)}\left(t_{e}\right)=0,
\end{array}\right\}
$$

under the constraints that $c_{1}$ and $c_{3}$ fullfil the same relations.

Following a similar procedure with the equation

$$
\left(a_{1} a_{2}-a_{3}\right) v^{3}+\left(a_{1}^{2}+a_{2}\right) v^{2}+2 a_{1} v+1=0,
$$

we can find that in the article by Górecki and Szymkat (1983) it is proved that the roots of the equation

$$
8 r^{3}+8 a_{1} r^{2}+2\left(a_{2}+a_{1}^{2}\right) r+a_{1} a_{2}-a_{3}=0
$$

are as follows:

$$
r_{1}=\frac{s_{1}+s_{2}}{2}, \quad r_{2}=\frac{s_{2}+s_{3}}{2}, \quad r_{3}=\frac{s_{3}+s_{1}}{2} .
$$

Setting $2 r=p$ in 35 , we obtain the equation

$$
p^{3}+2 a_{1} p^{2}+\left(a_{1}^{2}+a_{2}\right) p+a_{1} a_{2}-a_{3}=0 .
$$

whose roots are $p_{1}=s_{1}+s_{2}, p_{2}=s_{2}+s_{3}, p_{3}=s_{3}+s_{1}$. Let

$$
p=\frac{1}{q} .
$$

Then Eqn. (36) has the following form:

$$
\left(a_{1} a_{2}-a_{3}\right) q^{3}+\left(a_{1}^{2}+a_{2}\right) q^{2}+2 a_{1} q+1=0,
$$

and its roots are

$$
q_{1}=\frac{1}{s_{1}+s_{2}}, \quad q_{2}=\frac{1}{s_{2}+s_{3}}, \quad q_{3}=\frac{1}{s_{3}+s_{1}} .
$$

Finally, from (19) and (39), we obtain that

$$
\begin{aligned}
& \frac{x^{(1)}\left(t_{0}\right)}{x^{(2)}\left(t_{0}\right)}=\frac{c_{2}}{c_{3}}=\frac{1}{s_{1}+s_{2}}, \\
& \frac{x^{(1)}\left(t_{0}\right)}{x^{(2)}\left(t_{0}\right)}=\frac{c_{2}}{c_{3}}=\frac{1}{s_{2}+s_{3}}, \\
& \frac{x^{(1)}\left(t_{0}\right)}{x^{(2)}\left(t_{0}\right)}=\frac{c_{2}}{c_{3}}=\frac{1}{s_{3}+s_{1}} .
\end{aligned}
$$

Theorem 4. From the relation (40), it is possible to determine the zeros of $x\left(t_{0}\right)$ (if they exist) using the relations

$$
\left.\begin{array}{l}
x^{(1)}\left(t_{0}\right)\left(s_{1}+s_{2}\right)-x^{(2)}\left(t_{0}\right)=0, \\
x^{(1)}\left(t_{0}\right)\left(s_{2}+s_{3}\right)-x^{(2)}\left(t_{0}\right)=0, \\
x^{(1)}\left(t_{0}\right)\left(s_{3}+s_{1}\right)-x^{(2)}\left(t_{0}\right)=0,
\end{array}\right\}
$$

under the constraints that $c_{2}$ and $c_{3}$ fullfil the same relations.
A generalization of these result relations (33) and (41) to higher order equations may be obtained directly, due to the following remark.

Remark 1. The relations (33) and (41) may be obtained directly from the following propositions.

Let the coefficients $A_{i}$ of the solution $x(t)$ fullfil the relations

$$
\left.\begin{array}{lll}
A_{1}=0 & A_{2} \neq 0, & A_{3} \neq 0, \\
A_{2}=0 & A_{1} \neq 0, & A_{3} \neq 0, \\
A_{3}=0 & A_{1} \neq 0, & A_{2} \neq 0 .
\end{array}\right\}
$$

In this way, we obtain equations which contain only two exponential terms, and such equations can be solved in analytical form.

The relations (42) are more general than (33) and (41) because they are also valid when $c_{2} \neq 0$ or $c_{1} \neq 0$. Moreover, they also hold for higher order equations. For such equations, to obtain only two exponential terms, it is necessary to assume more than one coefficient $A_{i}$ equal to zero.

\section{Basic result}

Theorem 5. The equation

$$
x(t)=\sum_{i=1}^{n} A_{i} e^{s_{i} t}
$$

or

$$
x^{(1)}(t)=\sum_{i=1}^{n} s_{i} A_{i} e^{s_{i} t}
$$

can be decomposed into a system of equations containing a set of equations composed of only two terms. The set contains

$$
\left(\begin{array}{c}
n \\
n-2
\end{array}\right)=\frac{1}{2} n(n-1)
$$

equations with two exponential terms.

Example 4. For $n=3$, we have the following equations:

$$
\begin{aligned}
x(t) & =A_{1} e^{s_{1} t}+A_{2} e^{s_{2} t}+A_{3} e^{s_{3} t}, \\
x^{(1)}(t) & =A_{1} s_{1} e^{s_{1} t}+A_{2} s_{2} e^{s_{2} t}+A_{3} s_{3} e^{s_{3} t},
\end{aligned}
$$

where

$$
\begin{aligned}
& A_{1}=\frac{c_{3}-\left(s_{2}+s_{3}\right) c_{2}+s_{2} s_{3} c_{1}}{\left(s_{1}-s_{2}\right)\left(s_{1}-s_{3}\right)}, \\
& A_{2}=\frac{c_{3}-\left(s_{3}+s_{1}\right) c_{2}+s_{3} s_{1} c_{1}}{\left(s_{2}-s_{3}\right)\left(s_{2}-s_{1}\right)}, \\
& A_{3}=\frac{c_{3}-\left(s_{1}+s_{2}\right) c_{2}+s_{1} s_{2} c_{1}}{\left(s_{3}-s_{1}\right)\left(s_{3}-s_{2}\right)} .
\end{aligned}
$$

Looking for an extremum, we use Eqn. (46), where the necessary condition is $x^{(1)}(t)=0$. Assuming that

$$
x^{(1)}(t)=0, \quad A_{1}=0,
$$


after eliminating the initial condition $c_{1}$ from $(50)$, we obtain that

$$
e^{\left(s_{2}-s_{3}\right) t_{e}}=\frac{c_{3}-s_{2} c_{2}}{c_{3}-s_{3} c_{2}}
$$

where

$$
\begin{gathered}
e^{\left(s_{3}-s_{1}\right) t_{e}}=\frac{c_{3}-s_{3} c_{2}}{c_{3}-s_{1} c_{2}}, \\
e^{\left(s_{1}-s_{2}\right) t_{e}}=\frac{c_{3}-s_{1} c_{2}}{c_{3}-s_{2} c_{2}}, \\
c_{1}=\frac{1}{s_{2} s_{3}}\left[\left(s_{2}+s_{3}\right) c_{2}-c_{3}\right] .
\end{gathered}
$$

Similarly, asumming $A_{2}=0$, we obtain, after eliminating $c_{2}$, that

$$
\begin{aligned}
e^{\left(s_{2}-s_{3}\right) t_{e}} & =\frac{c_{3}-s_{2}^{2} c_{1}}{c_{3}-s_{3}^{2} c_{1}} \frac{s_{3}}{s_{2}}, \\
e^{\left(s_{3}-s_{1}\right) t_{e}} & =\frac{c_{3}-s_{3}^{2} c_{1}}{c_{3}-s_{1}^{2} c_{1}} \frac{s_{1}}{s_{3}}, \\
e^{\left(s_{1}-s_{2}\right) t_{e}} & =\frac{c_{3}-s_{1}^{2} c_{1}}{c_{3}-s_{2}^{2} c_{1}} \frac{s_{2}}{s_{1}} .
\end{aligned}
$$
obtain

Finally, asumming $A_{3}=0$, after eliminating $c_{3}$, we

$$
\begin{aligned}
e^{\left(s_{2}-s_{3}\right) t_{e}} & =\frac{c_{2}-s_{2} c_{1}}{c_{2}-s_{3} c_{1}} \frac{s_{3}}{s_{2}}, \\
e^{\left(s_{3}-s_{1}\right) t_{e}} & =\frac{c_{2}-s_{3} c_{1}}{c_{2}-s_{1} c_{1}} \frac{s_{1}}{s_{3}}, \\
e^{\left(s_{1}-s_{2}\right) t_{e}} & =\frac{c_{2}-s_{1} c_{1}}{c_{2}-s_{2} c_{1}} \frac{s_{2}}{s_{1}} .
\end{aligned}
$$

Similarly, the equation

$$
x(t)=A_{1} e^{s_{1} t}+A_{2} e^{s_{2} t}+A_{3} e^{s_{3} t}+A_{4} e^{s_{4} t}=0
$$

can be decomposed into the following set of equations:

$$
\begin{array}{llll}
A_{1} e^{s_{1} t}+A_{2} e^{s_{2} t}=0, \text { where } A_{3}=0 & \text { and } & A_{4}=0, \\
A_{1} e^{s_{1} t}+A_{3} e^{s_{3} t}=0, \text { where } A_{2}=0 & \text { and } & A_{4}=0, \\
A_{1} e^{s_{1} t}+A_{4} e^{s_{4} t}=0, \text { where } A_{2}=0 & \text { and } & A_{3}=0, \\
A_{2} e^{s_{2} t}+A_{3} e^{s_{3} t}=0, \text { where } A_{1}=0 & \text { and } & A_{4}=0, \\
A_{2} e^{s_{2} t}+A_{4} e^{s_{4} t}=0, \text { where } A_{1}=0 & \text { and } & A_{3}=0, \\
A_{3} e^{s_{3} t}+A_{4} e^{s_{4} t}=0, \text { where } A_{1}=0 & \text { and } & A_{2}=0 .
\end{array}
$$

It is a set of

$$
\left(\begin{array}{l}
4 \\
2
\end{array}\right)=\frac{3 \cdot 4}{2}=6
$$

equations with only two exponential terms.

Remark 2. It is evident that looking for $x\left(t_{0}\right)=0$ instead of $x^{(1)}\left(t_{e}\right)=0$, we must multiply the relations 51 - (59) a propriately by $s_{i} / s_{j}$. For example,

$$
e^{\left(s_{j}-s_{i}\right) t_{0}}=\frac{c_{3}-s_{j} c_{2}}{c_{3}-s_{i} c_{2}} \frac{s_{i}}{s_{j}}
$$

and so on.
Remark 3. If Eqn. (3) has repeated roots, then the relations (2) and (11) must be transformed by properly passing to the limit.

In the particular case, when $s_{1}=s_{2}=\cdots=s_{n}=s$, we obtain

$$
\begin{gathered}
x(t)=e^{s t} \sum_{k=1}^{n} A_{k} t^{k-1} \\
A_{k}=\sum_{i=0}^{k} \frac{x^{(k)}(0)(-1)^{i} s^{i}}{i !(k-i) !}, \quad k=1,2, \ldots, n .
\end{gathered}
$$

The necessary condition for the existence of the local extremum of the solution (2) is $x^{(1)}(t)=0$, and the problem is reduced to an algebraic one,

$$
\sum_{k=1}^{n} A_{k}\left[s t_{e}^{(k-1)}+(k-1) t_{e}^{k-2}\right]=0 .
$$

\section{Conclusion}

It was shown that every differential equation of the $n$-th order can be decomposed into a set of $\frac{1}{2} n(n-1)$ equations of the 2-nd order, which can be solved in analytical form.

\section{References}

Górecki, H. (2004). A new method for analytic determination of extremum of the transients in linear systems, Control and Cybernetics 33(2): 275-295.

Górecki, H. and Szymkat, M. (1983). Application of an elimination method to the study of the geometry of zeros of real polynomials, International Journal of Control 38(1): 1-26.

Górecki, H. and Turowicz, A. (1968). Determination of the dependence of the maximal deviation of the controlled quantity and the time of deviation on the parameters in linear control systems, Avtomatika i Telemekhanika (6): 179-181.

Górecki, H. (2009). A new method for analytic determination of extremum of the transients in linear systems, Bulletin of the Polish Academy of Sciences: Technical Sciences 57(2): 153-155.

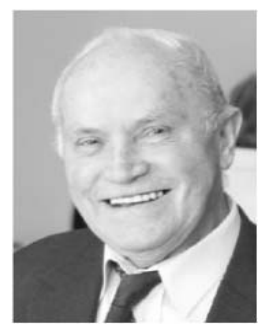

Henryk Górecki was born in Zakopane in 1927. He received the M.Sc. and Ph.D. degrees in technical sciences from the AGH University of Science and Technology in Cracow in 1950 and 1956, respectively. Since the beginning of his academic activity he has been attached to the Faculty of Electrical Engineering, Automatics and Electronics of the AGH University of Science and Technology. In 1972 he became a full professor and up to 1997 he was the director of the Institute of Automatics. He has lectured extensively in automatics, control theory, optimization and technical cybernetics. He is a pioneer of automatics in Poland as the author of the first book on this topic in the country, published in 1958. For many years he was the head of doctoral studies and the supervisor of $78 \mathrm{Ph} . \mathrm{D}$. students. He is the author or co-author of 20 books, and among them a monograph on control systems with delays in 1971, and about 200 scientific articles in international journals. His current research interests include optimal control of systems with time 
delay, distributed parameter systems and multicriteria optimization. Professor Górecki is an active member of the Polish Mathematical Society, the American Mathematical Society and the Committee on Automatic Control and Robotics of the Polish Academy of Sciences, a Life Senior Member of the IEEE, a member of technical committees of the IFAC as well as many Polish and foreign scientific societies. He was chosen a member of the Polish Academy of Arts and Sciences (PAU) in 2000. He was granted an honorary doctorate of the AGH University of Science and Technology in Cracow in 1997. He has obtained many scientific awards from the Ministry of Science and Higher Education as well as the award of the Prime Minister (2008), the Academy of Sciences and the Mathematical Society. He was honored with the Commander's Cross of the Order of Polonia Restituta in 1993.

Received: 21 January 2010

Revised: 7 April 2010 